\title{
Integer and Fractional Approaches of a Pair of Car-Following Models
}

\author{
Luis Enrique Abriz Morales', Oscar Alfonso Rosas Jaimes ${ }^{2}$ \\ ${ }^{1,2}$ Benemérita Universidad Autónoma de Puebla \\ 4 Sur 104 Colonia Centro, Puebla, Mexico \\ luis.abriz@alumno.buap.mx; oscar.rosasj@correo.buap.mx
}

\begin{abstract}
There exist models to represent vehicular traffic, most of them are represented by ordinary linear or nonlinear differential equations. One of such approaches is the well-known Gazis-Herman-Rothery (GHR) model, which is formed by two cars moving in a single line where the car in front acts like a disturbance to the car behind, if assuming that overtakings does not occur. In this paper we take the model already mentioned, one with a nonlinear nature which depends on positions and velocities of the involved cars, to make a comparison between the classical model and one with modifications on the degree of its derivative, changing it with to a fractional order one. A set of data was obtained directly from an experiment with vehicles and special instruments, which has been used to make simulations in order to test the level of approximation of these models to the real behaviour. Through the data obtained we can note that the fractional model does not take any advantage in contrast to the integer-degree GHR model, but this situation is different to other models.
\end{abstract}

Keywords: Car-following model, GHR model, Conformal Fractional Derivative, Fractional Calculus.

\section{Introduction}

On the last years different theories have been developed in relation to the vehicular traffic that take into account many characteristics and qualities of the traffic phenomena with appreciable results [1], furthermore, those theories have attracted the attention of many researchers with different points of view. A lot of this theories are still in development and of course far away to be perfect, however they have been cemented in a gratify way. One of this traffic theories is known as the followthe-leader theory or car-following theory [2]. This field of study represents car systems in a single line with the condition that one car cannot overtake the another which is in front. The theory is based on the fact that each driver has different driving habits and reactions to stimulus that could influence the driving process. This implies the existence of a leader car (L) (car in front) and the other behind called follower (f). The leader has the freedom to speed up or to decelerate, while the follower must react to such changes of speed, with due cautions. This process has different levels of precision, leading to distinct approaches and models. For example, it has been observed that not only the acceleration phase is relevant, but the deceleration too, being that there exist asymmetry characteristics [3] in driving phenomena.

In most of dynamic phenomena, with the time as an independent variable, equations describe features of systems represented by them. Traditionally, those equations have an integer order $\alpha$ on their derivatives, i.e. $\alpha=1$, corresponds to a first-order differential equation. However, fractional-order differential equations have been considered with a crescent frequency to achieve a better precision in modelling of the most diverse nature [4]. In spite of fractional-order derivatives and fractional-order differential equations are not a novelty [5], it has been until recently that they are taking importance among the mathematical and control community. Due to its abstract formulation, there is no only one definition to a fractional-order derivative, but many proposals [6]. We will be referring to the conformal fractional derivative [7] because, as it will be shown, this fractional derivative possesses many of the properties of the classical integer-order one [8].

On the other hand, many fractional models have been proposed for many different systems [4, 6, 9, 10 and 11]. In the particular case of this present work herein shown, GHR integer-order model has been adjusted through a step-wise optimization algorithm with the intention to study its approximation to a pair of experimental data sets of velocity. Later, the degree of this model's differential equation is modified to a fractional one. Leaving their parameters fix, its degree is adjusted by means of step-wise algorithm again. This is also performed for another simpler model, known as the Pipes' model. We then compare the performance of these two models in their integer form, with those in their fractional scheme. It will be 
shown that the Pipes' model is better in performance in its fractional form, but GHR has a better approach in its integerdegree differential equation.

\section{Description of models}

\subsection{Pipes' Model}

The first and an older car-following model is that proposed by L.A. Pipes, [12]. Pipes' model is very intuitive because it represents the acceleration $\dot{v}_{f}(t)$ of the follower car through the difference between the velocity of the car in front $v_{L}(t)$ and the car behind $v_{f}(t)$. This is described by the differential equation (1)

$$
\frac{d v_{f}(t)}{d t}=\lambda\left[v_{L}(t)-v_{f}(t)\right]
$$

Where $\lambda$ is the sensitivity parameter which describes driving habits of the follower car. Calibrating such a parameter [13] gives a value in the interval $\lambda \in(0,1]$, which physically signifies that values near to 0 indicates less reactive drivers and values tending to 1 is associated with more reactive drivers.

\subsection{GHR Model}

The Gazis-Herman-Rothery (GHR) model is probably the most studied car-following equation, basically, the theory is structured in the "response = sensitivity X stimulus" way. The GHR model assumes that the vehicle's acceleration is influenced by differences between the speeds and relative positions of the of cars involved. The GHR model [14] is a nonlinear model and it is defined as:

$$
\frac{d v_{f}(t)}{d t}=\frac{a v_{f}^{m}(t)\left[v_{L}(t)-v_{f}(t)\right]}{\left[x_{L}(t)-x_{f}(t)\right]^{l}}
$$

Where $v_{L}(t)$ is the leading car's velocity, $v_{f}(t)$ is the follower car's velocity, while $x_{L}(t)$ and $x_{f}(t)$ are their respective positions. If Eq. (2) and Eq. (3) are compared, it is possible to notice that

$$
\lambda=\frac{a v_{f}^{m}(t)}{\left[x_{L}(t)-x_{f}(t)\right]^{l}}
$$

i.e. this model considers that $\lambda$ is totally related to driving conditions and driver's habits [15]. Then, parameters $m$ and $l$ must be calibrated to reflect the specific features of each driver. The constant $a$ is included to make Eq. (2) dimension-consistent. One relevant characteristic of the GHR model is that one can obtain the Pipes' Model by making $m=0$ and $l=0$.

\section{Definitions}

Definition $2.1[7] f:[0, \infty) \rightarrow \mathbb{R}$ and $t>0$. Then the derivative definition of $f$ with respect to $t$ is:

$$
\frac{d f}{d t}=\lim _{\epsilon \rightarrow 0} \frac{f(t+\epsilon)-f(t)}{\epsilon}
$$

Is noticeable that Definition 2.1 pertains to the already known classical derivative or first-order derivative. The derivative can be used like an operator $D^{n}$, where $n \in \mathbb{N}$ is the derivative order. 
In the same way, it known that the classical derivative satisfy the following rules for $n=1, f=f(t)$ and $g=g(t)$ :

1) Derivative of a constant value: $D^{1}(\lambda)=0$, with $f(t)=\lambda$

2) Power rule: $D^{1}\left(t^{p}\right)=p t^{p-1}$

3) Product rule: $D^{1}(f g)=f D^{1}(g)+d D^{1}(f)$

4) Quotient rule: $D^{1} \frac{f}{g}=\frac{g D^{1}(f)-f D^{1}(g)}{g^{2}}$

5) Chain rule: $D^{1}(f o g)=D^{1}(f)(g(t)) D^{1}(g(t))$

6) Linearity: $D^{1}(a f+b g)=a D^{1}(f)+b D^{1}(g)$ for all $a, b \in \mathbb{R}$

When a fractional-order derivative is defined, the principal goal is to make a modification of $n \in \mathbb{N}$ to become $n=\alpha \in$ $\mathbb{Q}$ or $n=\alpha \in \mathbb{R}$.

One definition of fractional-order derivative [7] is proposed by Khalil et al. (2014), that was applied by them too, one peculiarity of this is that it also satisfies the rules already mentioned [8]. The following definition differed for most of the other definitions of fractional-order derivatives proposed until now [6].

Definition 2.2 Let $f:[0, \infty) \rightarrow \mathbb{R}$ and $t>0$. Thus the conformable fractional derivative, [8], with order $\alpha$ is defined by:

$$
D^{\alpha}(f)(t)=\lim _{\epsilon \rightarrow 0} \frac{f\left(t+\epsilon t^{1-\alpha}\right)-f(t)}{\epsilon}
$$

for $\alpha \in(0,1)$.

Many times, to denote the conformable fractional derivatives of $f$ of order $\alpha$, it is opportune to write $f^{\alpha}(t)$ like $D^{\alpha}(f)(t)$. Additionally, if the conformable fractional derivative of $f$ occurs, then it is possible to say that $f$ is $\alpha$ differentiable [8]. It is important to say that these derivative of order $\alpha$ complies the properties and rules mentioned previously.

To notice these rules and properties, it can see an important feature of the conformal fractional derivative, i.e. if $\alpha$ takes the value of 1 , the rules of classical derivatives are retrieved.

Definition 2.3 Let's consider the next fractional differential equations:

$$
y^{\alpha}(t)=f(t, y)
$$

with $y_{0}=y(0)$.

The following expression is obtained from replacing the definition of the conformable fractional derivative in equation (4):

$$
\lim _{\epsilon \rightarrow 0} \frac{y\left(t+\epsilon t^{1-\alpha}\right)-y(t)}{\epsilon}=f(t, y)
$$

If $\epsilon$ is considered small enough as to unmake the notation of limit then:

$$
\frac{y\left(t+\epsilon t^{1-\alpha}\right)-y(t)}{\epsilon}=f(t, y)
$$

Let $h=\epsilon t^{1-\alpha}$. Thus $\epsilon=\frac{h}{t^{1-\alpha}}=h t^{\alpha-1}$, so the equations (7) can be seen as: 


$$
\frac{y(t+h)-y(t)}{h t^{\alpha-1}}=f(t, y)
$$

If (8) is solved for $y(t+h)$, consequently the result is in the following expression

$$
\left.y(t+h)=y(t)+f(t, y) h t^{\alpha-1}\right)
$$

Thereafter, the last equation is the numerical method of Euler to solve differential equations but in the fractional version of this taking as reference the classical Euler's method to resolve differential equations of first-order, [16].

Definition 2.4 From equation (1) and from Definition 2.3 it is possible to obtain the next fractional-order equation:

$$
D^{\alpha} v_{f}(t)=\frac{a v_{f}^{m}\left[v_{L}(t)-v_{f}(t)\right]}{\left[x_{L}(t)-x_{f}(t)\right]^{l}}
$$

This fractional-order equation can be called the GHR fractional model.

\section{Experimental Data}

With the purpose to have a tool with which the data set gotten from the simulations of both models can be compared, an experiment was realized using two cars that they run in a close circuit (Figure 1). It was possible to get a pair of very similar vehicles with features as those listed in Table 1. The brand, version and the transmission type were considered in order to make these experiments as driver-dependent as possible, trying to avoid any other causes of discrepancy.

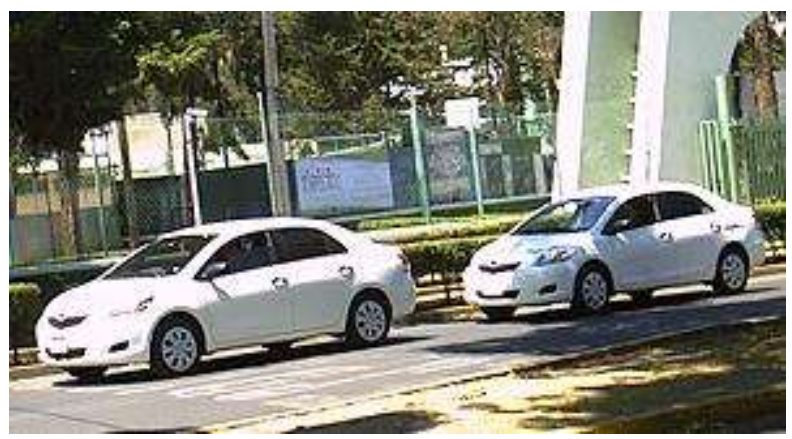

Fig. 1: Two-cars in pair.

Table 1: Data of vehicles and drivers.

\begin{tabular}{|ccccc|cc|}
\hline \multicolumn{5}{c}{ Vehicle } & \multicolumn{2}{c|}{ Driver } \\
\hline Car Company & Brand & Vehicle ID & Year & Transmission & Gender & Age \\
\hline Toyota & Yaris & 1 & 2010 & Manual & Man & 40 \\
Toyota & Yaris & 2 & 2010 & Manual & Man & 52 \\
\hline
\end{tabular}

The circuit which was run by the pair, had about $2.5 \mathrm{~km}$, located in urban street. It was chosen because it has traffic lights and bumps, but also by the safety and comfort of the participants into the experiment. 
Two trials were conducted (X and Y, Table 1). In a first trial $(\mathrm{X})$ car identify as 1 took the role of leader and car named 2 took the role of follower. Then, in a second trial (Y) car named as 2 was the leader, changing roles with car identified as 1 which performed as follower. In both cases the circuit already mentioned was fully covered. This experiment was conducted on a Sunday, in order to minimize the compromise to safety of the volunteers when interacting with other drivers. By the aid of a scanner for OBD (On-Board-Diagnostics) and suitable software, the velocities of both cars could be registered in the form of independent data sets. These were later synchronized and filtered, in order to know the speeds developed by the two vehicles at some precise moment. By means of knowing the initial positions of both vehicles, it was possible to estimate the relative distance between them, giving an estimation of the relative positions of each car.

It was asked to each driver to behave like they used to do, with two important restrictions:

a) Follower should not overtake leader.

b) Follower should not admit other car to be located between it and the leader, unless the situations turned in an unsafety one.

Table 2 presents the organization of the trials, where the number to each car given in the Table 1 indicates the role that both cars held.

Table 2: Organization of the trials.

\begin{tabular}{|cc|cc|}
\hline \multirow{2}{*}{ Trial } & \multirow{2}{*}{ Vehicle } & \multicolumn{2}{|c|}{ Car Number } \\
& & Leader & Follower \\
\hline $\mathrm{X}$ & \multirow{2}{*}{ Yaris } & 1 & 2 \\
$\mathrm{Y}$ & & 2 & 1 \\
\hline
\end{tabular}

Each vehicle had a co-pilot, who should comply with the task to manage the software hosted in a laptop computer which was connected to the OBD terminal of each car to obtain the data [17].

\section{Simulations}

Numerically the equation (1) can be solved for $v_{f}(t)$ by the classical method of Euler. The Euler fractional method was used to solve fractional equation (11) also for $v_{f}(t)$, this method is expressed by equation (10), all with the objective of compare both models and the experimental data collected from the experiment. It is important to mention that the results as much from classic Pipes' model (green line) as from fractional Pipes' model (cyan line) are obtained from [18], those values were replicated and used to make the comparison.

The velocity profile of the follower car got in the experiment is showed in the Figure 2 (blue line). To applicate the numerical method in both models, the velocities and positions of leader $\left(v_{L}(t)\right.$ and $x_{L}(t)$ respectively) are required, such requirements are not shown on this work. The results from the GHR classical model, which is from integer order, are illustrated in the red line. On the same way, the GHR fractional model (11) is used to calculate through the numerical method (10) the velocity of follower, generating a similar graphic, but not the same, its profile is shown in black.

There are some disadvantages in the numerical results when applied to these models due to some lack of accuracy of our data, especially when both cars were very near to each other, causing some few apparent overtakings of the followers over the leaders. This caused complex values, for example. Fortunately, this situations have been infrequent in our work, and the evolution of our simulations including those data sets are mostly well-behaved. To denote the precision of each model (classic and fractional GHR model) respect to the data obtained from the experiment and with the respective Pipes' model, an average of the absolute error is calculated. The equation (12) calculates the average absolute error between the measured data of the follower velocities $v_{f m}(t)$ and the velocities $v_{f c}(t)$ obtained by means of the classical numerical method of Euler for differential equations during the complete simulation, where $T$ is the total of data throughout the simulation. 


$$
e_{c}=\frac{\sum\left|v_{f m}(t)-v_{f c}(t)\right|}{T}
$$

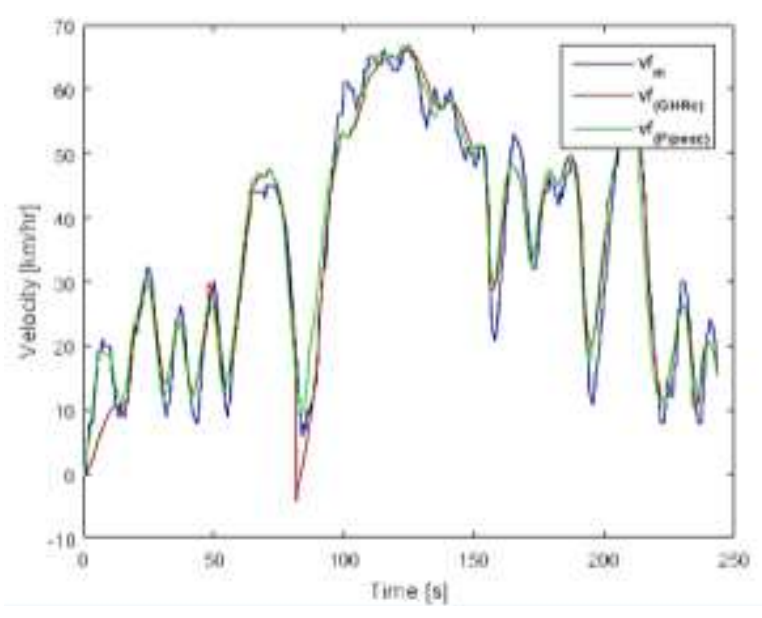

(a) Trial X Classic models

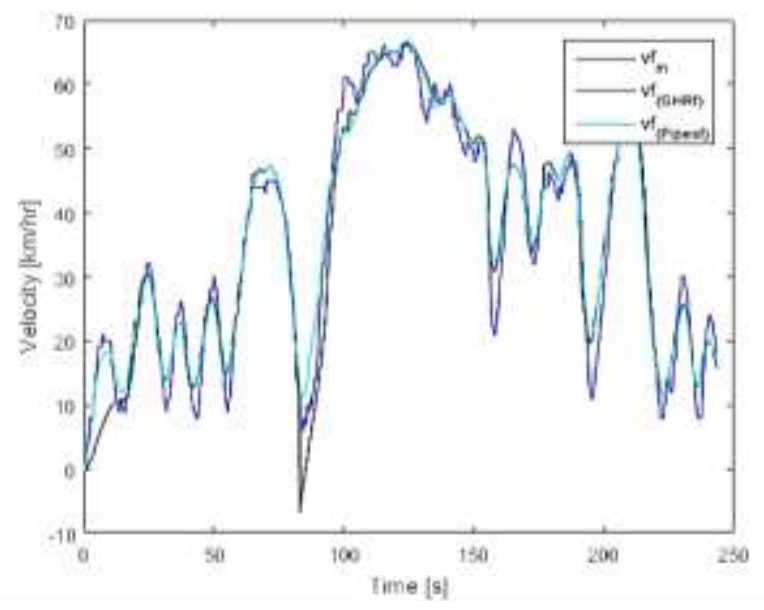

(b) Trial X Fractional models

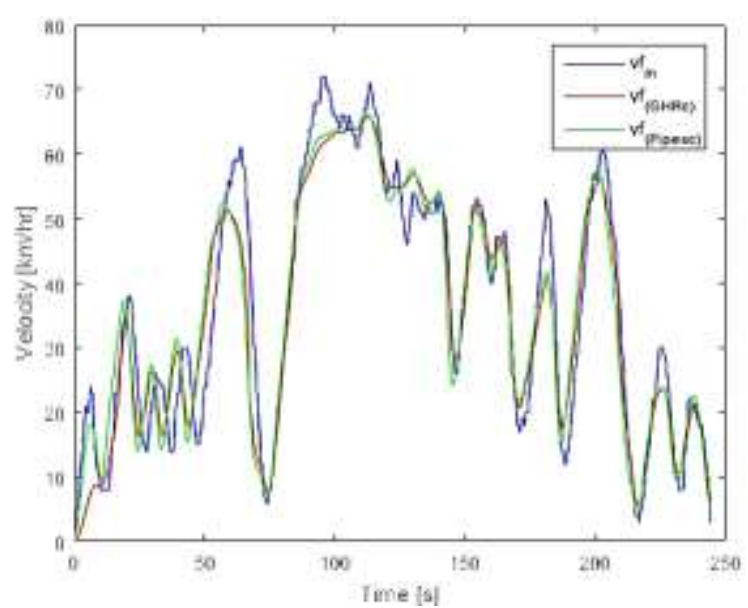

(c) Trial Y Classic models

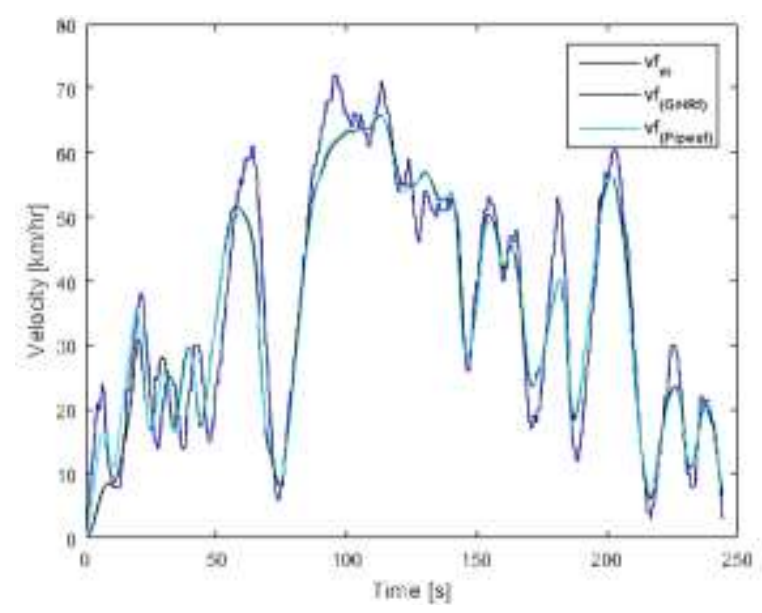

(d) Trial Y Fractional models

Fig. 2: Comparison of the velocity profile from the experiment versus the numerical solutions of GHR and Pipes models.

Equation (13) shows the way to get the error between the measured velocities $v_{f m}(t)$ and the follower velocities information $v_{f f}(t)$ obtained from the fractional numerical method of Euler to differential equations of fractional-order.

$$
e_{f}=\frac{\sum\left|v_{f m}(t)-v_{f f}(t)\right|}{T}
$$

The results collected to equations (12) and (13) are displayed on Table (2), the column Error (c) is the numerical variance to the GHR classical model, in the other hand, the column Error $(f)$ reports the numerical difference of the GHR fractional model. 
The $\alpha$ columns represent the order of the fractional derivative to each model that was used in the numerical method, a relevant characteristic of definitions 2.2 is that it is possible to return to the classic GHR model by doing $\alpha=1 \in \mathbb{N}$, the $\alpha$ value may differ for a measured data set different from this, due to it can be adjust to get the minimum error.It was mentioned that if $m=0$ and $l=0$, Pipes' car-following model [12] can be recovered, similarly it is easy to see that with the corresponding data in the experiment $\mathrm{F}$ of [18] it is possible to recover the classic as well as the fractional Pipes' models.

Table 2: Profile velocity error for each car-following model.

\begin{tabular}{|c|c|c|c|c|c|c|c|c|c|}
\hline \multirow{2}{*}{ Trial } & \multirow{2}{*}{ Vehicle } & \multicolumn{4}{|c|}{ GHR Model } & \multicolumn{3}{|c|}{ Pipes' Model } \\
\cline { 3 - 10 } & & $m$ & $l$ & $\alpha$ & Error (c) & Error (f) & $\alpha$ & Error (c) & Error (f) \\
\hline $\mathrm{X}$ & \multirow{2}{*}{ Yaris } & 0 & 0.513 & 0.999 & 2.8412 & 3.1393 & 0.982 & 3.0803 & 2.6700 \\
\hline $\mathrm{Y}$ & & 0.001 & 0.425 & 0.998 & 4.4075 & 4.5591 & 0.981 & 5.0009 & 4.4843 \\
\hline
\end{tabular}

The results found by means of the models as for classical as for fractional approaches for GHR and Pipes can look alike, such as they are showed in the Figure 3. Nevertheless, if we observed the error to each model, we can find that the classic GHR model is more precise than fractional Pipes' model. Although, the results obtained don't demonstrate the advantage of fractional calculus, they have a few similarities with the investigations that relate this aspects, see [9] for an example, the fractals and other events that are result of an unpredictable nature, such as habits and other aspects of driving.

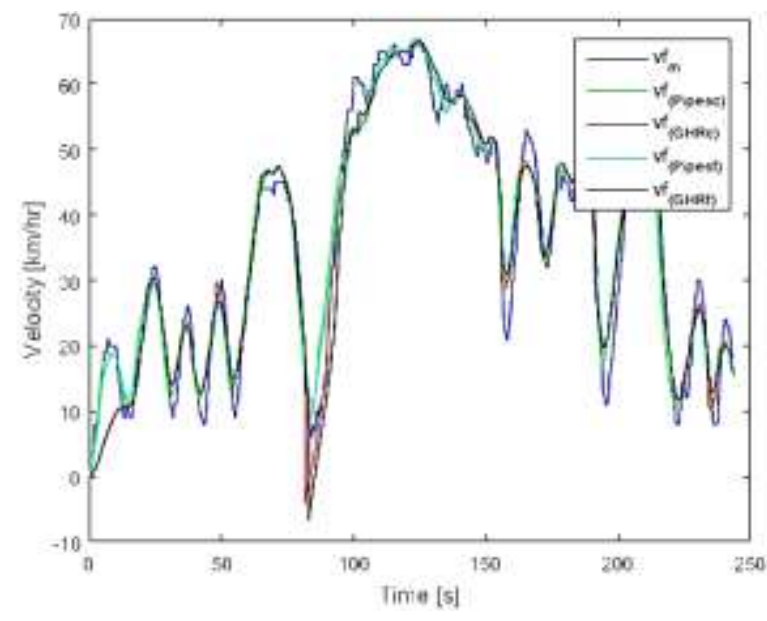

(e)

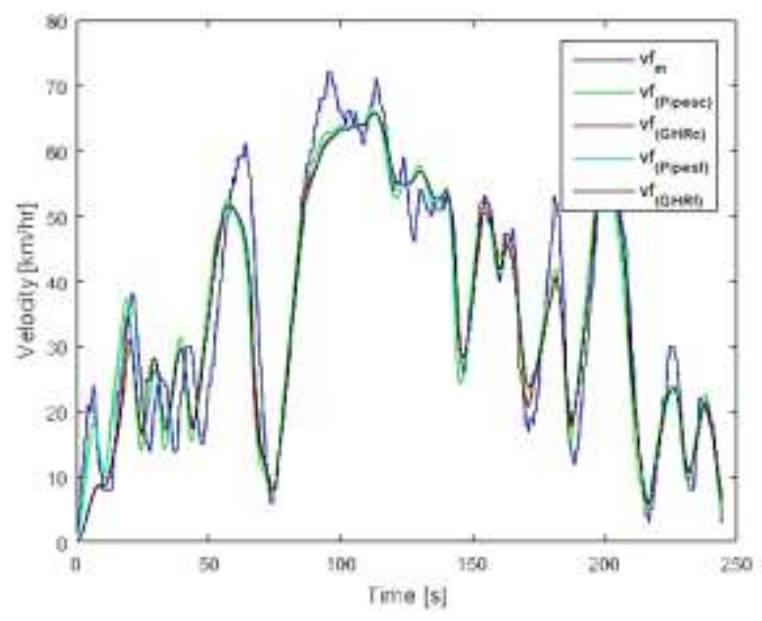

(f)

Fig. 3: Comparison of the velocity profiles from the experiment versus the all numerical solutions of GHR and Pipes models.

\section{Conclusion}

The conduct of two vehicles running in a single line can be represented through different dynamic models. In this paper, we have test two of them: Pipes' Model and GHR Model. Both are known as integral first-order differential equations in their formulation. Pipes takes into account a difference between leader and follower velocities $\left(v_{L}(t)-v_{f}(t)\right)$, while GHR takes not only such a difference, but a relation among relative positions $\left(x_{L}(t)-x_{f}(t)\right)$ and the velocity $v_{f}(t)$ of the car being perturbed by the variables. To perform a validation of such models some data were obtained from experiments directly performed on real cars under car-following conditions. Through the difference between the measured data and simulation results, we have obtained a comparison of the performance of these two models.

Additionally, we have taken the degrees of their respective derivatives appearing in their formulation and we have modified them into fractional order expressions. Once again, we performed some simulations trying to make a comparison 
of the performance of these approaches. It has been very interesting to find that the fractional-order Pipes model achieved a better approximation of its integer-order version. On the other hand, fractional-order GHR improved the performance of its integer-order version. These results can be analysed under the focus of the nature of each one of these schemes. The simple formulation of Pipes is useful for simple situations, but the nonlinear nature of GHR can be taken as a very accurate one. At the same time, the fractional-order versions add some valuable features when dealing to precision of different types of models.

The whole set of results obtained and reported in this document can be extended and be part of a more precise and better design set of experiments in order to get a more complete view of the accuracy of these models.

\section{References}

[1] Bevrani, Kaveh, Chung, Edward, \& Miska, Marc (2012) Evaluation of the GHR car following model for traffic safety studies. In Proceedings of the 25th ARRB Conference, ARRB Group Ltd, Perth, W. A., pp. 1-11.

[2] D.C. Gazis, R. Herman, R.W. Rothery, Nonlinear follow-the-leader models of traffic flow, Oper. Res. 9 (4) (1961) 545567.

[3] Li, X., Luo, X., He, M., \& Chen, S. (2018). An improved car-following model considering the influence of space gap to the response. Physica A: Statistical Mechanics And Its Applications, 509, 536-545. doi: 10.1016/j.physa.2018.06.069

[4] Uchaikin, V. V. (2013a). Fractional Derivatives for Physicists and Engineers: Volume I Background and Theory Springer Berlin Heidelberg.

[5] M.D. Ortigueira, Fractional Calculus for Scientists and Engineers. Springer, Dordrecht, Heidelberg etc. (2011); DOI: 10.1007/978-94-007-0747-7.

[6] Morita, T., \& Sato, K. (2013). Liouville and Riemann-Liouville fractional derivatives via contour integrals. Fractional Calculus And Applied Analysis, 16(3). doi: 10.2478/s13540-013-0040-9

[7] Khalil, R., Al Horani, M., Yousef, A., Sababheh, M. (2014) A new denition of fractional derivative J. Comput. Appl. Math., 264,pp. 65\{70\}

[8] Katugampola, U. N. (2011) A new fractional derivative with classical properties Journal of the American Math- ematical Society, arXiv: 1410.6535v2, 2011.

[9] Uchaikin, V. V. (2013b). Fractional Derivatives for Physicists and Engineers: Volume II Applications Springer Berlin Heidelberg.

[10] Zhao, D., Pan, X., \& Luo, M. (2018). A new framework for multivariate general conformable fractional calculus and potential applications. Physica A: Statistical Mechanics And Its Applications, 510, 271-280. doi: 10.1016/j.physa.2018.06.070

[11] Sun, H., Zhang, Y., Baleanu, D., Chen, W., \& Chen, Y. (2018). A new collection of real world applications of fractional calculus in science and engineering. Communications In Nonlinear Science And Numerical Simulation, 64, 213-231.doi: 10.1016/j.cnsns.2018.04.019

[12] Pipes, L.A. (1953). An operational analysis of the traffic dynamics. Journal of Applied Physics, 24, pp. 271-281.

[13] Rosas-Jaimes, O. A., Luckie-Aguirre, O. and Lopez Rivera, J. C. (2013) Identi_cation and Analysis of a Sensibility Parameter of a Microscopic Tra_c Model. Proceedings of the Road Safety and Simulation Conference, RSS 2013, October 23-25, Rome, Italy, pp. 99-102.

[14]D.C. Gazis, R. Herman, R.W. Rothery, Nonlinear follow-the-leader models of traffic flow, Oper. Res. 9 (4) (1961) 545567.

[15] L. Edie, "Car-Following and Steady-State Theory for Noncongested Traffic", Operations Research, vol. 9, no. 1, pp. 66-76, 1961. Available: 10.1287/opre.9.1.66.

[16] Mathews, J. H. \& Fink, K. D. (2001), Numerical Methods with MATLAB, 3rd edition Williams Publishing House, United States.

[17] Rosas-Jaimes, O. A., Luckie-Aguirre, O. \& López-Rivera, J. C. (2014) Calibration and Comparison of Two Micrsocopic Traffic Models. Proceedings of the Latinamerican Congress of Automatic Control, CLCA 2014, Cancun, Mexico, pp. 1006-1011. 
[18] Rosas-Jaimes, Oscar \& Quezada Téllez, Luis Alberto \& Fernandez-Anaya, G. (2017). A Fractional Approach to Carfollowing Pipe's Model. Proceedings Congreso Nacional de Control Automático 2017 Monterrey, Nuevo León, Mexico, Octubre 4-6, 2017 